\title{
Integrable Nonlinear Equations and Liouville's Theorem, I
}

\author{
L. A. Dickey \\ Leningradsky av. 28 fl. 59125040 Moscow, USSR
}

\begin{abstract}
A symplectic structure for stationary Lax equations of the type $[L, P]=0$ is constructed, where $L$ is a matrix differential operator of the first order. It is shown that the equation has a sufficient for the complete integrability amount of first integrals in involution. The well-known linearization of the equation by the Abelian mapping is obtained in a natural manner in consequent exercising of Liouville's procedure.
\end{abstract}

This paper continues a series of works by Gelfand and the author $([2-4]$ and others) which deal with the equations of the Lax type $L_{t}=[P, L]$. Here $P, L$ are differential operators such that the order of $[P, L]$ is less than that of $L$. Novikov noticed that the stationary variants of these Lax equations are equally interesting. These stationary variants are obtained by assuming that $P, L$ are independent of $t$, i.e. equations of the form $[P, L]=0$ (sometimes called the Novikov equations). They are ordinary differential equations, totally integrable in a pure classical sense, i.e. solvable by quadratures. Since then the theory has branched into two parts: nonstationary and stationary. They often come into contact. For example, if there are two commuting Lax equations (e.g. two higher $\mathrm{KdV}$ equations) then the set of all solutions of the stationary problem for one of them is an invariant (and finite dimensional) manifold for the other. In such a way solitons and periodic finite-zonal solutions can be obtained. Nevertheless the nonstationary theory and the stationary one are quite different, with different problems and methods.

Gelfand and the author in their joint work were guided by an idea that it is the Hamiltonian structure of the equations under consideration which plays the leading role. The fact that the $\mathrm{KdV}$ equation is one of the Hamiltonian types was first established by Gardner, Zakharov and Faddeev; Gelfand and the author have given the general construction of Lax equations for an arbitrary order of the operator $L$, scalar as well as matrix (another construction was given by Kritchever) and proved that these equations are Hamiltonian. Moreover, they have constructed explicitly the Hamiltonian structure of the equations. Since then this structure has been studied by many authors. The greatest progress was achieved by Adler, Manin and Lebedev who have given a transparent group explanation of this Hamiltonian structure. Let us also mention works by Kuperschmidt and Wilson, 
Semenov-Tjan-Shanskii and Reyman, Drienfeld and Sokolov. We emphasize that we are speaking here about the nonstationary equations.

The Hamiltonian structure of the stationary equations has been studied much less. As stated earlier these equations can be solved by quadrature. This has been done using algebraic geometry methods by Novikov and Dubrovin, McKean and Moerbeke, Matveev and Its for the stationary KdV equations, and by Dubrovin and Kritchever for the general case. On the other hand these equations were obtained from the very beginning in a variational, Lagrangian form. Therefore it was clear that they were Hamiltonian systems and it was only natural to connect the fact of their integrability with the existence of a large number of first integrals in involution, i.e. with the Liouville theorem. The construction of first integrals and the proof of their involutiveness was given in [2-4]. However, a gap remained between the proof of the existence of first integrals in involution and effectively carrying out the Liouville integration procedure and obtaining the solution formulas. To that end an explicit construction of the Hamiltonian structure was needed. This was done for the $\mathrm{KdV}$ equation in [1] and we turn now to the general case.

Thus, in the present paper the following is done. An explicit expression for the Hamiltonian structure (the symplectic form and the Hamiltonian) for the stationary equations is given and the Liouville integration is carried out (i.e. the "actionangle" variables are obtained). The Abel mapping of a Riemann surface onto its Jacobi variety comes into existence by itself instead of being brought in from the outside. The same regular integration process explains the sense of special variables (proposed by Dubrovin); those are the variables in which the symplectic form splits up. Thus, this paper contains not new results (except for explicit formulas for the Hamiltonian structure), but rather a new interpretation of the integration process, on a basis of the Hamiltonian structure.

The first part of this article deals with equations created by a first order matrix differential operator $L$. This part is closely connected with works by Dubrovin $[5,6]$. It was highly stimulating for us to think over his works. In the second part an $n^{\text {th }}$ order differential operator $L$ (scalar, for simplicity) will be considered. This part is formally connected with works by Kritchever, however, it is considerably less dependent on them than the first part is on Dubrovin's works, as we use other variables. We apply a method of reducing $n^{\text {th }}$ order differential equations to sets of first order equations; in other words, we imbed the variety of $n^{\text {th }}$ order operators $L$ as a submanifold into the variety of matrix first order operators. Nowadays this method is widely used, e.g. by Kuperschmidt and Wilson [7], Drienfeld and Sokolov $[8]$, but only in connection with nonstationary problems.

The main tool in our search for a symplectic structure for Lagrangian equations will be the following general fact pointed out in [2]. If there is a Lagrangian $\mathscr{L}$ which polynomially depends on variables $u_{i}$ and their derivatives $u_{i}^{\prime}, u_{i}^{\prime \prime}, \ldots$, then the symplectic form corresponding to the Lagrangian set of equations $\delta \mathscr{L} / \delta u_{i}=0$ can be found in such a way. The variation $\delta \mathscr{L}=\sum_{i, k} \frac{\partial \mathscr{L}}{\partial u_{i}^{(k)}} \delta u_{i}^{(k)}$ should be transformed by parts: 


$$
\delta \mathscr{L}=\sum_{i, k}(-1)^{k}\left(\frac{\partial \mathscr{L}}{\partial u_{i}^{(k)}}\right)^{(k)} \delta u_{i}+\frac{d}{d x} \omega=\sum_{i} \frac{\delta \mathscr{L}}{\delta u_{i}} \delta u_{i}+\frac{d}{d x} \omega,
$$

where $\omega$ is a differential 1-form, i.e. a linear combination of $\delta u_{i}^{(k)}$. Then $\Omega=\delta \omega$ will be just the symplectic form corresponding to the set of equations $\frac{\delta \mathscr{L}}{\delta u_{i}}=0$. The Hamiltonian $H$ can be found from an equation $d H / d x=-\sum u_{i}^{\prime} \delta \mathscr{L} / \delta u_{i}$.

This article immediately follows the paper under the same title by two authors [1] (though formally is independent of it). Both authors of [1] realized that such a study was necessary.

1. We start with the equation

$$
R^{\prime}+[U+\zeta A, R]=0 .
$$

Here $R, U$ and $A$ are $n \times n$ matrices; $A$ is a diagonal matrix with elements $a_{i}=$ const $\neq 0, a_{i} \neq a_{j}(i \neq j)$, and $U$ is a matrix with arbitrary elements $u_{i j}, u_{i i}=0$. Further we consider the differential algebra $\mathscr{A}$ of polynominals in $u_{i j}$, and $u_{i j}^{\prime}$, $u_{i j}^{\prime \prime}, \ldots$ with complex coefficients. The assertion that an identity holds in $\mathscr{A}$ is equivalent to the assertion that it holds when the letters $u_{i j}, u_{i j}^{\prime}, \ldots$ are replaced by arbitrary functions $u_{i j}(x)$ and their derivatives. The equation expresses the fact that the differential operator of the first order $L=\partial+U+\zeta A(\partial=d / d x)$ commutes with $R$. The matrix $R$ is a solution of (1) we are looking for. We seek the solutions as a formal series

$$
R=\sum_{k=0}^{\infty} R_{k} \zeta^{-k}, R_{k, i j} \in \mathscr{A} .
$$

(Note that eq.(1) is satisfied by $R=\left(\varphi_{i} \psi_{j}\right)$, where $\varphi=\left\{\varphi_{i}\right\}$ and $\psi$ are solutions of $L \varphi=0$ and of the adjoint equation respectively.)

Solutions (2) form a ring (if two matrix functions $R_{1}$ and $R_{2}$ commute with $L$, so does their product $R_{1} R_{2}$ ).

2. We shall say that an element of $\mathscr{A}$ does not contain a constant if the free term of the corresponding differential polynomial is equal to zero.

Proposition. If all $R_{k, i j}$ in solution (2) do not contain a constant, this solution is a trivial zero.

Proof. Suppose that the assertion does not hold. Without loss of generality we may assume that $R_{0} \neq 0$. Equation (1) is equivalent to a recurrent formula

$$
R_{k}^{\prime}+\left[U, R_{k}\right]=-\left[A, R_{k+1}\right], k=-1,0,1,2, \ldots
$$

For $k=-1$ we have $\left[A, R_{0}\right]=0$. Hence $R_{0}$ is a diagonal matrix. For $k=0$ the diagonal terms of the equality yield $R_{0}^{\prime}=0, R_{0}=$ const, and under our assumption all the constants must be equal to zero.

Corollary. The solutions are uniquely determined by their constants.

3. In the sequel we shall construct special solutions $R^{\alpha}, \alpha=1, \ldots, n$ for which

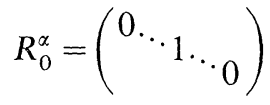


with unity in the $\alpha^{\text {th }}$ place and no other constants.

Proposition. The equations

$$
R^{\alpha} R^{\beta}=\delta_{\alpha \beta} R^{\alpha}, \sum_{\alpha=1}^{n} R^{\alpha}=I
$$

hold.

This means that $R^{\alpha}$ are projection operators forming a spectral decomposition of unity.

Proof. The proposition is a direct consequence of Sect. 2 since $R^{\alpha} R^{\beta}$ is a solution without constants if $\alpha \neq \beta$ and with the same constants as in $R^{\alpha}$ if $\alpha=\beta$

4. From (3) we can find

$$
\begin{aligned}
& R_{0, j k}^{\alpha}=\delta_{\alpha j} \delta_{\alpha k}, \\
& R_{1, j k}^{\alpha}=\frac{\delta_{\alpha j}-\delta_{\alpha k}}{a_{j}-a_{k}} u_{j k}, \\
& R_{2, j k}=\left\{\begin{array}{l}
-\frac{\delta_{\alpha j}-\delta_{\alpha k}}{\left(a_{j}-a_{k}\right)^{2}} u^{\prime}{ }_{j k}-\sum_{\beta \neq j, k} \frac{u_{j \beta} u_{\beta k}}{a_{j}-a_{k}}\left(\frac{\delta_{\alpha j}-\delta_{\alpha \beta}}{a_{j}-a_{\beta}}-\frac{\delta_{\alpha \beta}-\delta_{\alpha k}}{a_{\beta}-a_{k}}\right), \quad j \neq k \\
\sum_{\beta \neq j} \frac{\delta_{\alpha \beta}-\delta_{\alpha j}}{\left(a_{\beta}-a_{j}\right)^{2}} u_{j \beta} u_{\beta j}, \quad j=k .
\end{array}\right.
\end{aligned}
$$

5. Proposition. The general form of the solution is

$$
R=\sum_{\alpha=1}^{n} w_{\alpha}(\zeta) R^{\alpha}
$$
where $w_{\alpha}(\zeta)$ are the formal series $\sum_{r=r_{0}}^{\infty} w_{\alpha, r} \zeta^{-r} ; w_{\alpha, r}=$ const; $w_{\alpha}(\zeta)$ are the roots of
the characteristic equation

$$
\operatorname{det}(R-w I)=0 .
$$

Proof. From (3) it really follows that in $R_{k}$ constants may occur only in diagonal terms. By properly choosing the series $w_{\alpha}(\zeta)$ we can construct a solution with an arbitrary combination of constants in diagonal terms, i.e. an arbitrary solution

6. Now we consider the equation

$$
\varphi^{\prime}+(U+\zeta A) \varphi=\lambda \varphi
$$

where $\varphi$ is a column vector.

Proposition. There exists a solution of (7) of the form

$$
\begin{aligned}
\varphi=\sum_{r=0}^{\infty} \varphi_{r} \zeta^{-r}, \lambda=\sum_{r=-1}^{\infty} \lambda_{r} \zeta^{-r}, \quad \varphi_{r}=\left(\varphi_{r, k}\right), k=1, \ldots, n ; \\
\varphi_{r, k}, \lambda_{r} \in \mathscr{A},
\end{aligned}
$$


normalized by the condition $\varphi_{0, k}=\delta_{\alpha, k}, \alpha$ being an arbitrary integer, $\alpha=1, \ldots, n$; $\lambda_{-1}=a_{\alpha}$.

Proof. Let us put $\varphi_{r, \alpha}=0$ for $r>0$. Equation (7) is equivalent to

For $k=\alpha,(8)$ degenerates into

$$
\varphi_{r, k}^{\prime}+\left(U \varphi_{r}\right)_{k}=-a_{k} \varphi_{r+1, k}+\sum_{s=-1}^{\infty} \lambda_{s} \varphi_{r-s, k}, k=1, \ldots, n .
$$

$$
\left(U \varphi_{r}\right)=\lambda_{r}, \quad(r \geqq 0) .
$$

For $r=-1, k=\alpha$, relation (8) means that $\lambda_{-1}=a_{\alpha}$. For any $r \geqq 0$, we obtain $\lambda_{r}$ from (9) and then $\varphi_{r+1, k}(k \neq \alpha)$ from (8)

We denote this solution as $\varphi^{\alpha}, \lambda^{\alpha}$. Let $\Phi$ be the matrix $\left(\varphi_{k}^{\alpha}\right)$ where $\alpha$ is the number of a column and $k$ is that of a row. Then

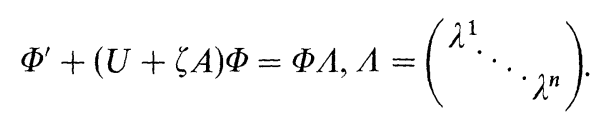

The matrix $\Phi=\sum_{r=0}^{\infty} \Phi_{r} \zeta^{-r}$ has the inverse $\Psi=\Phi^{-1}$, in the class of a formal series, because $\Phi_{0}=I$. It can easily be verified that

$$
-\Psi^{\prime}+\Psi(U+\zeta A)=\Lambda \Psi .
$$

If $\Psi=\left(\psi_{\alpha}^{j}\right)$ where $j$ is the number of a column and $\alpha$ is that of a row, then the row vector $\psi_{\alpha}$ satisfies the equation

$$
-\psi^{\prime}+\psi(U+\zeta A)=\lambda \psi
$$

the adjoint equation of (7).

Remark. The introduction of $\lambda$ in (7) and (11) made it possible to obtain a solution such that $\varphi_{r, k} \in \mathscr{A}$; otherwise it would be necessary to extent the algebra by adding the operation of integration.

7. Proposition. The matrix

$$
R^{\alpha}=\varphi^{\alpha} \psi_{\alpha}=\left(\varphi_{i}^{\alpha} \psi_{\alpha}^{j}\right)
$$

is the projection operator from 3.

Proof. The matrix $R^{\alpha}$ satisfies (1). This follows from (7) and (12). Moreover, it can be easily seen that $R_{0}^{\alpha}$ is of the required form (see Sect. 3) and that there are no other constants in $R_{k}^{\alpha}$

8. Proposition. tr $R^{\alpha}=1$.

Proof. Taking the traces of both sides of (1) we see that $\operatorname{tr} R=$ const for all solutions. As for $R^{\alpha}$, it contains constants only in the term $R_{0}^{\alpha}$, and $\operatorname{tr} R_{0}^{\alpha}=1$

9. Now the variations of the variables $u_{j k}^{(l)}$ will be introduced. Let us denote them as $\delta u_{j k}^{(l)}$. We consider the differential forms $\sum a \delta u_{j k}^{(l)} \wedge \delta u_{p q}^{(l)} \wedge \ldots$ The operator $\delta$, the covariant derivative acts in the usual way, e.g. $\delta f=\sum \partial f / \partial u_{j k}^{(l)} \cdot \delta u_{j k}^{(l)}$. 
Proposition. The relation

$$
\delta \operatorname{tr} A R^{\alpha}=\operatorname{tr}\left(R_{\zeta}^{\alpha} \delta U\right)+\partial \operatorname{tr}\left(\delta \varphi^{\alpha} \psi_{\alpha, \zeta}-\varphi_{\zeta}^{\alpha} \delta \psi_{\alpha}\right),(\partial=d / d x)
$$

holds.

(The subscript $\zeta$ denotes the derivative with respect to $\zeta$.)

Proof. We start with Eqs. (7) and (12) and apply $\delta$ to them.

$$
\begin{gathered}
\delta \varphi^{\prime}+(U+\zeta A) \delta \varphi+\delta U \varphi=\lambda \delta \varphi+\delta \lambda \cdot \varphi \\
-\delta \psi^{\prime}+\delta \psi(U+\zeta A)+\psi \delta U=\lambda \delta \psi+\delta \lambda \cdot \psi .
\end{gathered}
$$

Next we differentiate (7) and (12) with respect to $\zeta$ :

$$
\begin{gathered}
\varphi_{\zeta}^{\prime}+(U+\zeta A) \varphi_{\zeta}+A \varphi=\lambda \varphi_{\zeta}+\lambda_{\zeta} \varphi, \\
-\psi_{\zeta}^{\prime}+\psi_{\zeta}(U+\zeta A)+\psi A=\lambda \psi_{\zeta}+\lambda_{\zeta} \psi .
\end{gathered}
$$

Now we multiply (15a) by $\psi_{\zeta}$ on the right and $(15 \mathrm{~b})$ by $\varphi_{\zeta}$ on the left, add them together and take the trace. We transform some terms:

$$
\begin{aligned}
& \operatorname{tr} \delta \varphi^{\prime} \psi_{\zeta}-\operatorname{tr} \varphi_{\zeta} \delta \psi^{\prime}=\partial\left(\operatorname{tr} \delta \varphi \cdot \psi_{\zeta}\right)-\operatorname{tr}\left(\delta \varphi \cdot \psi_{\zeta}^{\prime}\right)-\partial \operatorname{tr} \varphi_{\zeta} \delta \psi \\
& +\operatorname{tr} \varphi_{\zeta}^{\prime} \delta \psi=\partial \operatorname{tr}\left(\delta \varphi \cdot \psi_{\zeta}-\varphi_{\zeta} \delta \psi\right)-\operatorname{tr}\left\{\delta \varphi \left[\psi_{\zeta}(U+\zeta A)+\psi A\right.\right. \\
& \left.\left.-\lambda \psi_{\zeta}-\lambda_{\zeta} \psi\right]+\left[(U+\zeta A) \varphi_{\zeta}+A \varphi-\lambda \varphi_{\zeta}-\lambda_{\zeta} \varphi\right] \delta \psi\right\}
\end{aligned}
$$

(from (16)). Altogether we have

$$
\begin{aligned}
& \partial \operatorname{tr}\left(\delta \varphi \cdot \psi_{\zeta}-\varphi_{\zeta} \delta \psi\right)-\operatorname{tr}(\delta \varphi \cdot \psi+\varphi \delta \psi) A+\operatorname{tr} \delta U\left(\varphi \psi_{\zeta}+\varphi_{\zeta} \psi\right) \\
& +\lambda_{\zeta} \operatorname{tr}(\delta \varphi \cdot \psi+\varphi \delta \psi)-\delta \lambda \operatorname{tr}\left(\varphi \psi_{\zeta}+\varphi_{\zeta} \psi\right)=0 .
\end{aligned}
$$

We have $\operatorname{tr}(\delta \varphi \cdot \psi+\varphi \delta \psi)=\operatorname{tr} \delta(\varphi \psi)=\operatorname{tr} \delta R^{\alpha}=0$ since $\operatorname{tr} R^{\alpha}=1$. The term containing $\delta \lambda$ vanishes for the same reason. The remaining terms imply the required statement

10. Further we deal with equations of Lagrangian type

$$
\frac{\delta \mathscr{L}}{\delta v_{i}}=0, i=1, \ldots, N,
$$

where $\mathscr{L}$ is a differential polynomial in some variables $v_{i}, i=1, \ldots, N ; \delta / \delta v_{i}$ are variational derivatives

$$
\frac{\delta}{\delta v_{i}}=\sum_{k=0}^{\infty}(-\partial)^{k} \frac{\partial}{\partial v_{i}^{(k)}}
$$

We shall consider the case when the Eqs. (17) are the same order, say $p$, with respect to each variable $v_{i}$. More precisely, the highest derivatives $v_{i}^{(p)}$ will enter linearly with constant coefficients:

$$
\begin{aligned}
& \frac{\delta \mathscr{L}}{\delta v_{1}}=a_{11} v_{1}^{(p)}+\ldots+a_{1 N} v_{N}^{(p)}+\ldots \\
& \frac{\delta \mathscr{L}}{\delta v_{N}}=a_{N 1} v_{1}^{(p)}+\ldots+a_{N N} v_{N}^{(p)}+\ldots
\end{aligned}
$$


Moreover, for the case under consideration the condition $\operatorname{det}\left(a_{i j}\right) \neq 0$ will hold, which will enable us to express the highest derivatives in terms of lower ones. The phase space is Np-dimensional, with independent variables $v_{i}^{(j)}, j<p$. The vector field $\xi$ connected with equation (17) acts on functions defined in the phase space as the operator $\partial$ with subsequent elimination of $v_{i}^{(p)}$ appearing in differentiation, with the help of (17).

11. It is known that for every $\mathscr{L} \in \mathscr{A}$ the variation $\delta \mathscr{L}$ can be decomposed uniquely into a sum

$$
\delta \mathscr{L}=\sum A_{i} \delta v_{i}+\partial \omega,
$$

where $\omega$ is a 1-form; $\partial$ acts on forms in the following way: it differentiates both the coefficients and the variations $\delta v_{i}^{(k)}, \partial \delta u_{i}^{(k)}=\delta u_{i}^{(k+1)}$. The coefficients $A_{i}$ turn out to be equal to $\delta \mathscr{L} / \delta v_{i}$ (see [2]).

Another well-known fact we use here (see[2]) is as follows: there exists a differential polynomial $H\left(v_{i}^{(k)}\right), k<p$ such that

$$
\partial H=-\sum v_{i}^{\prime} \frac{\delta \mathscr{L}}{\delta v_{i}} .
$$

Proposition (2). If we put $\omega^{(2)}=\partial \omega$ then the relation

holds.

$$
\delta H=-i(\partial) \omega^{(2)}-\sum_{i} \frac{\delta \mathscr{L}}{\delta v_{i}} \delta v_{i}
$$

Here $i(\partial)$ symbolizes the usual substitution of a vector field in a 2 -form.

Proof. The formula can be checked by applying $\partial$ to both sides of the equation and using (19), (20) ( $\delta$ and $\partial$ commute)

From this proposition it follows that $\delta H=-i(\xi) \omega^{(2)}$ where $\xi$ is a vector field connected with Eq. (17) (see Sect. 10). This means that the equation has the Hamiltonian form with respect to the symplectic form $\omega^{(2)}$ and the Hamiltonian $H$. This method of obtaining the Hamiltonian structure was suggested in [2] and used in [1] for the $\mathrm{KdV}$ equation.

Further the elements $u_{i j}$ of the matrix $U$ will play the role of $v_{i}$. Let $\delta \mathscr{L} / \delta U$ be the matrix with elements $\delta \mathscr{L} / \delta u_{i j}$. The diagonal elements of this matrix remain indefinite.

\section{Proposition 1.}

$$
\frac{\delta \operatorname{tr} A R^{\alpha}}{\delta U}={ }^{t}\left(\frac{\partial R^{\alpha}}{\partial \zeta}\right) \text {; that is } \frac{\delta \operatorname{tr} A R_{k+1}^{\alpha}}{\delta U}=k^{t} R_{k}^{\alpha}
$$

where the left superscript $t$ denotes the transposition of the matrix; the equality makes sense only for the nondiagonal terms.

Proof. The assertion immediately follows from (14)

Remark. "Variational theorem" (22) plays the main role in all works by Gelfand and the author. This theorem uses only the first term in (14). Reference [1] allows us also to make use of the second term. 
Let us take the Lagrangian

$$
\mathscr{L}=\sum_{\alpha} b_{\alpha} \operatorname{tr} A R_{m+2}^{\alpha}, \quad b_{\alpha}=\text { const } \neq 0, b_{\alpha} \neq b_{\beta}(\alpha \neq \beta) .
$$

From (22) we find that Eq. (17) has the form $\sum_{\alpha} b_{\alpha} R_{m+1}^{\alpha}=0$ (for the nondiagonal terms), that is

$$
\left[A, R_{m+1}\right]=0, R=\sum_{\alpha} b_{\alpha} R^{\alpha} .
$$

From (3) it is easily seen that the highest derivatives contained in $\left(\sum_{\alpha} b_{\alpha} R_{m+1}^{\alpha}\right)_{i j}$ are

$$
(-1)^{m+1} \frac{b_{i}-b_{j}}{\left(a_{i}-a_{j}\right)^{m+1}} u_{i j}^{(m)}
$$

This means that the matrix $a_{i j}$ of $(18)$ is a diagonal one and $\operatorname{det}\left(a_{i j}\right) \neq 0$. The set of Eqs. (24) has the order $m n(n-1)$.

Proposition 2. The form $\omega$ for the Hamiltonian system represented by the set of equations (24) is

$$
\omega=\left.(m+1) \sum_{\alpha=1}^{n} b_{\alpha} \operatorname{tr}\left(\varphi^{\alpha} \delta \psi_{\alpha}\right)\right|_{m+1} .
$$

The subscript $m+1$ indicates that the coefficient in $\zeta^{-m-1}$ should be taken.

Proof. According to (14) and Sect. 10 we have $\omega=\left.\sum b_{\alpha} \operatorname{tr}\left(\delta \varphi^{\alpha} \psi_{\alpha, \zeta}-\varphi_{\zeta}^{\alpha} \delta \psi_{\alpha}\right)\right|_{m+2}=$

$$
\left.\sum b_{\alpha}\left\{\delta \operatorname{tr}\left(\varphi^{\alpha} \psi_{\alpha, \zeta}\right)-\operatorname{tr}\left(\varphi^{\alpha} \delta \psi_{\alpha, \zeta}+\varphi_{\zeta}^{\alpha} \delta \psi_{\alpha}\right)\right\}\right|_{m+2}=\left.\delta \sum b_{\alpha} \operatorname{tr}\left(\varphi^{\alpha} \psi_{\alpha, \zeta}\right)\right|_{m+2}-\sum b_{\alpha} \frac{\partial}{\partial \zeta}
$$
$\left.\operatorname{tr}\left(\varphi^{\alpha} \delta \psi_{\alpha}\right)\right|_{m+2}$. The first term may be omitted since it is an exact differential and cannot play any role because the form $\omega^{(2)}=\partial \omega$ does not depend on it. Thus

$$
\omega=-\left.\frac{\partial}{\partial \zeta} \sum_{\alpha} b_{\alpha} \operatorname{tr}\left(\varphi^{\alpha} \delta \psi_{\alpha}\right)\right|_{m+2}=\left.(m+1) \sum_{\alpha} b_{\alpha} \operatorname{tr}\left(\varphi^{\alpha} \delta \psi_{\alpha}\right)\right|_{m+1} .
$$

13. We transform (25) in such a way that $\omega$ is expressed in terms of $R^{\alpha}$ instead of $\varphi^{\alpha}, \psi_{\alpha}$. If we add the exact differential (which can always be done) $\sum b_{\alpha} \delta \varphi_{j}^{\alpha} / \varphi_{j}^{\alpha}$ where $j$ is an arbitrary integer $\leqq n$ we obtain

$$
\omega=\left.\operatorname{tr}(m+1) \sum_{\alpha=1}^{n} b_{\alpha} \frac{\varphi^{\alpha}}{\varphi_{j}^{\alpha}} \delta\left(\varphi_{j}^{\alpha} \psi_{\alpha}\right)\right|_{m+1}=\operatorname{tr}(m+1) \sum_{\alpha=1}^{n} b_{\alpha} \frac{\varphi^{\alpha} \psi_{\alpha, \ell}}{\varphi_{j}^{\alpha} \psi_{\alpha, \ell}}
$$

$\left.\delta\left(\varphi_{j}^{\alpha} \psi_{\alpha}\right)\right|_{m+1}$ that is

$$
\omega=\left.(m+1) \sum_{\alpha=1}^{n} b_{\alpha} \frac{\left(\delta R^{\alpha} \cdot R^{\alpha}\right)}{R_{j l}^{\alpha}} j l\right|_{m+1},
$$

where $j, l$ are chosen arbitrarily.

14. Proposition. The Hamiltonian of the system under consideration is

$$
H=(m+1) \operatorname{tr}\left(A R_{m+2}+U R_{m+1}\right)=\left.(m+1) \operatorname{tr}(\zeta A+U) R\right|_{m+1} .
$$


Proof. It is sufficient to show (Sect. 11) that

$$
\partial H=-\operatorname{tr} U^{\prime}\left(\frac{\delta \mathscr{L}}{\delta U}\right)=(m+1) \operatorname{tr} U^{\prime} R_{m+1} .
$$

We check this by using (3) directly:

$$
\begin{aligned}
& \partial H=(m+1) \operatorname{tr} U^{\prime} R_{m+1}+\left.(m+1) \operatorname{tr}(\zeta A+U) R^{\prime}\right|_{m+1} \\
& =(m+1) \operatorname{tr} U^{\prime} R_{m+1}-\left.(m+1) \operatorname{tr}\left[(\zeta A+U)^{2}, R\right]\right|_{m+1}=(m+1) \operatorname{tr} U^{\prime} R_{m+1}
\end{aligned}
$$

Note that $H$ contains the phase variables only $u_{i j}^{(k)}, k<m$ only; the higher derivatives can be cancelled out from both terms.

We shall omit the factor $(m+1)$ both in $H$ and $\omega$.

15. Now we denote

$$
\widetilde{R}=\sum_{k=0}^{m} R_{k \zeta}^{m-k}
$$

Using (3) it can be shown that

$$
\tilde{R}^{\prime}+[U+\zeta A, \tilde{R}]=-\left[A, R_{m+1}\right] .
$$

Proposition. If in $R_{k}$ we substitute the derivatives of some function $u_{i j}(x)$ for the letters $u_{i j}^{(k)}$ then $\widetilde{R}$ satisfies $(1)$ if and only if $U(x)$ satisfies (24).

Proof. Obvious from (29)

16. Proposition. The coefficients of the polynomials in $\zeta$ :

$$
\operatorname{tr} \widetilde{R}^{k}, \quad k=1, \ldots, n
$$

are first integrals of (24). In this way we obtain $m n(n-1) / 2$ nontrivial first integrals. Proof. If $\tilde{R}$ satisfies (1) so does $\tilde{R}^{k}$. The trace of any solution is a constant, and hence $\operatorname{tr} \widetilde{R}^{k}=$ const, $\partial \operatorname{tr} R^{k}=0$.

Let us calculate how many nontrivial (i.e. nonconstant in the whole phase space) first integrals we have. Note that $\operatorname{tr} \tilde{R}$ gives only trivial first integrals since all its coefficients coincide with those of $\operatorname{tr} R$ which are identically constants. Among the $m k+1$ coefficients of $\operatorname{tr} \widetilde{R}^{k}$ the highest $m+1$ coefficients coincide with those of $\operatorname{tr} R^{k}$ and are therefore trivial. So we have $m+2 m+\ldots+(n-1) m=$ $\frac{n(n-1)}{2} m$ nontrivial first integrals which can be shown to be generically indipendent. The independence of the constructed first integrals has not been proved here (nor did Dubrovin, who introduced those integrals first). It is almost obvious that every next first integral contains a new variable which proves the independency.

Remark. Instead of (30) we can take as the first integrals the coefficients of the characteristic polynomial

$$
f(w, \zeta)=\operatorname{det}(\tilde{R}-w I)=\sum_{l=0}^{n} J_{l}(\zeta) w^{l}=\sum_{l=0}^{n} \sum_{k=0}^{(n-1) m} J_{k l} \zeta^{k} w^{l} .
$$

17. For any point $U, U^{\prime}, \ldots, U^{(m-1)}$ of the phase space the equation $f(w, \zeta)=0$ 
specifies an algebraic function. The main properties of this function were studied by Dubrovin. For the presentation to be self-contained we repeat here all the facts we need further.

The asymptotics of $w(\zeta)$ for $\zeta \rightarrow \infty$ can be obtained by comparing $\widetilde{R}$ with $R$. The $m+1$ highest terms of $\widetilde{R}$ coincide with those of $\zeta^{m} R$. Hence $m+1$ terms of the asymptotics of $w(\zeta)$ for $\zeta \rightarrow \infty$ coincide with those of the asymptotics of an eigenvalue of $\zeta^{m} R$. The eigenvalues of $\zeta^{m} R$ are $\zeta^{m} b_{\alpha}$, hence we have

\section{Proposition 1.}

$$
w(\zeta)=\zeta^{m} b_{\alpha}+0\left(\zeta^{-1}\right)
$$

The number $\alpha$ depends on the sheet of the Riemann surface. We denote by $\{\alpha\}$ the point $\zeta=\infty$ on the sheet where the asymptotics is $\zeta^{m} b_{\alpha}$.

Proposition 2. The number of branch points (generally, they are of second order) is $m n(n-1)$.

Proof. The calculation may be carried out with the help of the discriminant $\Delta=\prod_{i \neq j}\left(w_{i}-w_{j}\right)$. It is symmetrical with respect to $\left\{w_{i}\right\}$ and hence it can be expressed in terms of $\zeta$. Its asymptotics is $\zeta^{m n(n-1)}$ for $\zeta \rightarrow \infty$. Hence it has $m n(n-1)$ roots. These roots are the branch points of the Riemann surface

Proposition 3. The genus of the Riemann surface is $\rho=\frac{m n(n-1)}{2}-n+1$.

Proof. This follows from the usual formula for the genus: $2 \rho=\sum\left(j_{k}-1\right)-2 n+2$ where $j_{k}$ is the degree of the branch point

In what follows $P$ will denote the point of the Riemann surface.

18. The spectral projection operator of $R$ corresponding to an eigenvalue $w$ is given by the formula

$$
g(P)=\left(f_{w}\right)^{-1} \sum_{l=1}^{n} J_{l}(\zeta) \sum_{k=0}^{l-1} w^{k} \tilde{R}^{l-1-k} .
$$

Thus $g(P)$ is a polynomial in $\tilde{R}$. Therefore if $\tilde{R}$ satisfies (1) then so does $g(P)$.

When $P \rightarrow\{\alpha\}$ we have $g(P)=\left(\begin{array}{llll}0 & \cdots & & \\ & & \cdots & \\ & & & 0\end{array}\right)+0\left(\zeta^{-1}\right)$ (where unity stands in the $\alpha^{\text {th }}$ place). Moreover, the first $m+1$ terms of the asymptotics of $g(P)$ coincide with those of $R^{\alpha}(\zeta)$ (which is a projection operator of $R$ ),

$$
g(P)=R^{\alpha}(\zeta)+0\left(\zeta^{-m-1}\right) .
$$

We can even show that the remainder in $(34)$ is $0\left(\zeta^{-\infty}\right)$, that is the whole asymptotics coincide; this however should be understood in the sense that the higher derivatives in $R^{\alpha}$ must be expressed in terms of the phase variables $U, U^{\prime}, \ldots, U^{(m-1)}$ with the help of (24). This is true since both $g(P)$ and $R^{\alpha}$ satisfy $(1)$ and $g^{2}=g,\left(R^{\alpha}\right)^{2}=R^{\alpha}$ and these conditions uniquely define a formal series in $\zeta$.

Note that the elements of $g(P)$ are rational in $w$, i.e. they are rational functions 
on an algebraic curve. They have poles where $f_{w}=0$, i.e. at the branch points.

19. Proposition 1. The divisor of zeros of the function $g_{j i}$ in the finite part of the Riemann surface consists of two parts $d_{j}+d^{l}$ depending on the numbers $j$ and $l$ respectively.

Proof. The matrix $g$ is a one-dimensional projection operator and in a neighbourhood of a point different from branch points it can be written as $\left\{\tilde{\varphi}_{i}(P) \tilde{\psi}_{j}(P)\right\}$ where $\tilde{\varphi}_{i}, \tilde{\psi}_{j}$ are regular. The rest is obvious

From (5) we can see that at the point $\zeta=\{i\}$ the function $g_{j l}(j \neq l)$ has a double zero if $i \neq j, l$ and simple zeros if $i=j, l$. Thus we have $2(n-2)+2=2 n-2$ zeros at infinity. If $j=l$ then $g_{j j}$ in $\{i\}$ does not vanish when $i=j$ and has a double zero when $i \neq j$, altogether $2(n-1)$ zeros. We have shown that $g_{j i}$ has always $2(n-1)$ zeros at infinity.

Proposition 2. $g_{j l}$ has $m n(n-1)-2(n-1)$ zeros in the finite part of the Riemann surface.

Proof. The numbers of poles and of zeros of an algebraic function are equal. The function $g_{j l}$ has $m n(n-1)$ poles and $2(n-1)$ zeros at infinity

Proposition 3. If $\left|d_{j}\right|$ is the degree of the divisor $\left|d_{j}\right|$ then

$$
\left|d_{j}\right|=\left|d^{l}\right|=\frac{m n(n-1)}{2}-(n-1) .
$$

Proof. First of all $\left|d_{j}\right|$ does not depend on $j$. Indeed, $g_{j l} / g_{k l}$ has $d_{j}-d_{k}$ as its divisor of zeros and poles in the finite part of the Riemann surface. At infinity it has equal numbers of zeros and poles. Hence $\left|d_{j}\right|=\left|d_{k}\right| ;\left|d^{l}\right|$ does not depend on $l$ either. Now let us take into account that the Riemann surface and all the functions $g_{j l}$ depend on the point $U, U^{\prime}, \ldots, U^{(m-1)}$ of the phase space, and the dependence is analytical. This implies that $\left|d_{j}\right|$ must be constant throughout the whole phase space except, possibly, a submanifold of a complex codimension 1 (or a real codimension 2) where $\left|d_{j}\right|$ may be less. On the other hand, $R_{k}$ at a point ${ }^{t} U,-{ }^{t} U,{ }^{t} U$, $-{ }^{t} U, \ldots$ coincides with ${ }^{t} R_{k}$ at the point ${ }^{t} U,-{ }^{t} U,{ }^{t} U,-{ }^{t} U, \ldots$ (since if $R(U(x))$ is a solution of $(1)$ then so is $\left.{ }^{t} R\left({ }^{t} U(-x)\right)\right)$. Hence $g$ at a point $U, U^{\prime}, U^{\prime \prime}, \ldots$ is equal to ${ }^{t} g$ at ${ }^{t} U,-{ }^{t} U^{\prime},{ }^{t} U^{\prime \prime}, \ldots$ The divisor $d_{j}$ of the former is $d^{j}$ of the latter. Thus $\left|d_{j}\right|=$ $\left|d^{j}\right|$ as was stated

20. Besides the involution $U, U^{\prime}, U^{\prime \prime}, \ldots \mapsto{ }^{t} U,-{ }^{t} U^{\prime},{ }^{t} U^{\prime \prime}, \ldots$ the phase space admits an $n-1$ parameter transformation group preserving Eq. (24), the first integrals and the Hamiltonian structure. These are the transformations $\mu U \mu^{-1}$ where $\mu$ is an arbitrary constant diagonal matrix. Let us factorize the phase space with respect to this transformation group. The dimension of the factorized space is $m n(n-1)-(n-1)$. We shall diminish the dimension still more by restricting our space to invariant submanifolds $J_{k l}=$ const, $l=1, \ldots, n-1 ; k$ is the greatest number of a nontrivial first integral with given $l: k=(n-l) m-(m+1)=$ $(n-l-1) m-1$. The whole space is now stratified into these $m n(n-1)-$ 
$2(n-1)=2 \rho$ dimensional invariant phase subspaces. We shall integrate the equation in each of them. Now there remain $\frac{m n(n-1)}{2}-(n-1)=\rho$ first integrals. Note that the number of integrals is equal to half the dimension.

21. Now we rewrite expression (26) for the 1 -form using asymptotics (32), (34). We see that

$$
\omega=\left.\sum_{\alpha} w(P) \frac{(\delta g(P) \cdot g(P))_{j l}}{g_{j l}(P)}\right|_{1},(P \rightarrow\{\alpha\})
$$

The subscript 1 denotes the coefficient in $\zeta^{-1}$. We have obtained the sum of the residues of the differential

$$
w(P) \frac{(\delta g(P) \cdot g(P))_{j l}}{g_{j l}(P)} d \zeta
$$

at infinity. It may be replaced by the sum of the residues in the finite part of the Riemann surface:

$$
\omega=\sum_{\text {(poles) }} \operatorname{Res} w(P) \frac{(\delta g(P) \cdot g(P))_{j l}}{g_{j l}(P)} d \zeta .
$$

Theorem. 1-form connected with Hamiltonian equation (24) may be written as

where $\zeta_{P}$ is the $\zeta$-projection of $P$.

$$
\omega=-\sum_{P \in d_{j}} w(P) \delta \zeta_{P}
$$

Proof. The only poles of the differential in (35) are the points of the divisor $d_{j}$ since the points of $d^{l}$ can be cancelled out from the numerator and the denominator and the poles of $g$ (branch points are not the poles of the differential like the pole of $\frac{1}{\sqrt{z}}$ is not a pole of $\frac{1}{\sqrt{z}} d z$ ). Let $P^{*}$ be a point of $d_{j}$. We calculate the residue at this point. In the neighbourhood of this point it is convenient to write $g_{i k}$ as $\varphi_{i} \psi_{k}$ where $\sum \varphi_{i} \psi_{i}=1$ and $\varphi_{i}$ has zeros in $d_{i}, \psi_{k}$ in $d^{k}$. Then

$$
\begin{gathered}
\operatorname{Res} w(P) \frac{(\delta g(P) \cdot g(P))_{j l} d \zeta_{P=P^{*}}=\left.w\left(P^{*}\right) \operatorname{Res} \frac{\delta \varphi_{j}}{\varphi_{j}}\right|_{P=P^{*}}}{g_{j l}(P)} \\
=w\left(P^{*}\right) \operatorname{Res} \frac{\delta\left[\left(\zeta-\zeta_{P^{*}}\right) \varphi_{j}\right]}{\left(\zeta-\zeta_{P^{*}}\right) \varphi_{j}}=-w\left(P^{*}\right) \delta \zeta_{P^{*}}
\end{gathered}
$$

22. Now we remind the reader of Liouville's procedure for integrating Hamiltonian systems (for greater detail see [1]). Let us consider the submanifolds $M$ where $J_{k l}=$ const. $J_{k l}$ are in involution if and only if $M$ are Lagrangian submanifolds; this means that 1 -form $\omega$ is an exact differential $\delta V$ of a function defined on $M$ and depending on the parameter $J_{k l}$. On taking $J_{k l}$ and some quantities $\mu_{i}$ as coordinates in the phase space we obtain an expression for $\omega$ in the form

$$
\omega=\sum_{i}\left(\partial V / \partial \mu_{i}\right) \delta \mu_{i}+\sum_{k, l} a_{k l} \delta J_{k l}=\delta V+\sum_{k, l}\left(a_{k l}-\partial V / \partial J_{k l}\right) \delta J_{k l} .
$$


Now we put $\theta_{k l}=a_{k l}-\partial V / \partial J_{k l}$. Then $\omega=\delta V+\sum \theta_{k l} d J_{k l}$ and $\omega^{(2)}=\sum \delta \theta_{k l} \wedge \delta J_{k l}$. Thus $\theta_{k l}$ are coordinates canonically conjugate to $J_{k l}$. The Hamiltonian system can now be written as $\theta_{k l}^{\prime}=\partial H / \partial J_{k l} ; J_{k l}^{\prime}=-\partial H / \partial \theta_{k l}$ whence it follows that the solutions are

$$
\theta_{k l}=\frac{\partial H}{\partial J_{k l}} x+\theta_{k l}^{0}, \quad J_{k l}=J_{k l}^{0},
$$

where $\theta_{k l}^{0}, J_{k l}^{0}$ are arbitrary constants. The quadratures appear in this procedure when we find $V$.

Now we turn our attention to our case. The manifold $M$ where all $J_{k l}$ are constant is characterized by the property that for all its points, $f(w, \zeta)$ is the same and hence so is the function $w(\zeta)$ as well as its Riemann surface. In expression (36) for 1 -form there are differentials $\delta \zeta_{p *}$ but not the differentials $\delta J_{k l}$ (i.e. in the above procedure all $a_{k l}$ vanish). The quantities $\zeta_{P^{*}}$ (where $P^{*} \in d_{j}$ ) will be chosen as the additional coordinates $\mu_{i}$ mentioned above. These coordinates were introduced by Dubrovin. They appear here when we write 1-form $\omega$.

It is obvious that $\omega$ is integrable on the manifold $M$ because it proves to be a form with separable variables. The function $V$ is

$$
V=-\sum_{P^{*} \in d_{j}} \int^{*} w(P) d \zeta_{P}
$$

(here the lower limit of integration is arbitrary). From the integrability of the 1-form it follows that the first integrals $J_{k l}$ are in involution. Let us find the "angle" variables $\theta_{k l}$ :

$$
\theta_{k l}=-\sum_{P^{*} \in d_{j}} \int^{P_{j}^{*}} \frac{w(P)}{J_{k l}} d \zeta=\sum_{P^{*} \in d_{j}} \int^{P^{*}} \frac{\zeta^{k} w^{l}}{f_{w}} d \zeta
$$

Here we have Abel integrals of the first kind, i.e. the integrals of differentials holomorphic on the whole Riemann surface. The regularity at infinity must be checked. The behaviour of the integrand at infinity is

$$
\frac{\zeta^{k} w^{l}}{f_{w}} \sim \frac{\zeta^{k} w^{l}}{w^{n-1}} \sim \frac{\zeta^{k} \zeta m l}{\zeta^{m(n-1)}}=\zeta^{k+m(l-n+1)} .
$$

Since $k \leqq(n-1+1) m-2$, the exponent is not greater than -2 . On the righthand side of (38) we have the Abel mapping of the divisor $d_{j}$ into the Jacobi variety of the Riemann surface, i.e. the torus obtained by factorization of $\mathbb{C}^{\rho}$ with respect to the $2 \rho$-dimensional lattice of periods of the Abel integrals of the first kind. Thus we have

Theorem. The angle variables $\theta_{k l}$ corresponding to the action variables $J_{k l}$ can be obtained by the Abel mapping of the divisor $d_{j}$ (with arbitrary $j$ ).

Later we shall derive the expression of $H$ in terms of $J_{k l}$ and thus solution (37) of our set of equations. Note that the number $j$ was chosen arbitrarily. We could choose another number and find another solution. In (37) $\partial H / \partial J_{k l}$ does not depend on this choice but the constants corresponding to the different $j$ must be connected, otherwise there will be too many constants. The connection between the Abel 
mappings of two divisors $d_{j}$ with different $j$ (and of divisors $d^{i}$ ) was obtained by Dubrovin with the help of the Abel theorem: the Abel mapping of the divisor of a meromorphic function on the algebraic surface is zero. Let $\mathfrak{A}(d)$ be the Abel mapping of a divisor $d$. Let $d_{w}$ be the divisor of the branch points. The application of the Abel theorem to the function $g_{j i}$ gives

$$
\mathfrak{U}\left(d_{j}\right)+\mathfrak{A}\left(d^{i}\right)-\mathfrak{U}\left(d_{w}\right)+\mathfrak{U}\left(2 \sum_{\alpha}\{\alpha\}-\{j\}-\{i\}\right)=0,
$$

which yields the necessary connection. From this formula we also find

$$
\mathfrak{U}\left(d_{j}\right)-\mathfrak{A}(\{j\})=\mathfrak{U}\left(d_{k}\right)-\mathfrak{U}(\{k\}) .
$$

If the lower limit of integration in (37) is chosen as $\{j\}$ then $\mathfrak{U}(\{j\})=0$ and $\theta_{k l}^{0}, J_{k l}^{0}$ are independent of $j$.

It remains to return to the old variables. The functions $g_{i j}$ must be reconstructed from the Abel mapping of the divisors $d_{i}$ and $d^{j}$. Then $u_{i j}$ will be determined from the asymptotics (5). Thus, the Jacobi problem of inversion of the Abel mapping should be solved. Corresponding formulas were obtained by Dubrovin; we refer the reader to [6].

24. To find $\partial H / \partial J_{k l}$ it remains to express $H$ in terms of $J_{k l}$. Something more will be done. Another set of first integrals, including $H$, will be written and the connection between both sets of first integrals will be found. The new set of integrals is particularly interesting because of its connection with the theory of the nonstationary equation

$$
\dot{U}=\left[A, R_{m+1}\right] \text {. }
$$

It is easy to see that $\operatorname{tr} \int R_{k} d x$ are first integrals of this equation. Taking into account (22) it means that

$$
\operatorname{tr} \int R_{k}^{\alpha}\left[A, R_{m+1}\right] d x=0,
$$

that is $\operatorname{tr} R_{k}^{\alpha}\left[A, R_{m+1}\right]=\partial F_{k}^{\alpha}$, where $F_{k}^{\alpha} \in \mathscr{A}$ are some functions which are obviously first integrals of (24). From the general theory [2-4] it follows that the first integrals $\operatorname{tr} \int R_{k} d x$ are in involution with respect to the Hamiltonian structure for the nonstationary equation and that this implies the involutiveness of the first integrals in the stationary theory. (At present this is not of great importance to us: we shall express these first integrals in terms of $J_{k l}$ which are known to be in involution. In another context this might serve as a proof of involutiveness of $J_{k l}$ without an explicit calculation of the symplectic structure.)

Let us show that the two sets of the first integrals are linearly connected with each other. Let us put $L_{k, m+1}^{\alpha}=\operatorname{tr} R_{k}^{\alpha}\left[A, R_{m+1}\right]$. Then

$$
\begin{aligned}
& L_{k, m+1}=\operatorname{tr} R_{k}^{\alpha}\left(-R_{m}^{\prime}-\left[U, R_{m}\right]\right)=-\partial \operatorname{tr} R_{k}^{\alpha} R_{m}-\operatorname{tr}\left(-R_{k}^{\alpha^{\prime}} R_{m}-\left[U, R_{k}^{\alpha}\right] R_{m}\right) \\
& =-\partial \operatorname{tr} R_{k}^{\alpha} R_{m}-\operatorname{tr}\left[A, R_{k+1}^{\alpha}\right] R_{m}=-\partial \operatorname{tr} R_{k}^{\alpha} R_{m}+\operatorname{tr} R_{k+1}^{\alpha}\left[A, R_{m}\right] \\
& =-\partial \operatorname{tr}\left(R_{k}^{\alpha} R_{m}+R_{k+1}^{\alpha} R_{m-1}+\ldots+R_{k+m}^{\alpha} R_{0}\right)=-\left.\operatorname{tr}\left(R^{\alpha} \tilde{R}\right)\right|_{k} .
\end{aligned}
$$

The subscript $k$ denotes, as usual, the coefficient in $\zeta^{-k}$. We have $F_{k}^{\alpha}=$ 
$-\left.\operatorname{tr}\left(R^{\alpha} \tilde{R}\right)\right|_{k}$. We can replace $\tilde{R}$ by $\sum_{\beta} w_{\beta}(\zeta) R^{\beta}$ in this expression (since they have one and the same asymptotics). Thus we have

Proposition 1. The first integrals $F_{k}^{\alpha}$ are equal to the nontrivial coefficients of the asymptotics of $w_{\alpha}$, that is of the roots of the characteristic equation with coefficients $J_{k l}$ :

$$
F_{k}^{\alpha}=-w_{\alpha, k} .
$$

The coefficients of the equation, $J_{k l}$ and the coefficients $w_{\alpha, k}$ of the expansion of the roots into formal series are connected linearly.

Proposition 2.

$$
H=\sum_{\alpha=1}^{n} a_{\alpha} w_{\alpha, 2} .
$$

Proof. We know that $\partial H=-\operatorname{tr} U^{\prime} R_{m+1}$. Let us put $R^{*}=\sum a_{\alpha} R^{\alpha}$. This is another solution of (1) for which $R_{0}^{*}=A, R_{1}^{*}=U$ and $U^{\prime}+\left[A, R_{2}^{*}\right]=0$. We have

$$
\begin{aligned}
\partial H & =-\operatorname{tr} U^{\prime} R_{m+1}=\operatorname{tr}\left[A, R_{2}^{*}\right] R_{m+1}=\sum_{\alpha} \operatorname{tr} a_{\alpha}\left[A, R_{2}^{\alpha}\right] R_{m+1} \\
& =-\sum a_{\alpha} \operatorname{tr} R_{2}^{\alpha}\left[A, R_{m+1}\right]=-\partial \sum a_{\alpha} F_{2}^{\alpha}=\partial \sum a_{\alpha} w_{\alpha, 2}
\end{aligned}
$$

It remains to give the expression of $H$ in terms of $J_{k l}$. Put

$$
w_{\alpha}=b_{\alpha} \zeta^{m}+p_{\alpha} \zeta^{-1}+q_{\alpha} \zeta^{-2}+\ldots
$$

The equation $f(w, \zeta)=0$ can be written as $f_{0}+f_{m+1}+f_{m+2}+\ldots=0$ where

$$
\begin{aligned}
& f_{0}=\sum_{l=0}^{n} J_{l m, n-l} \zeta^{l m} w^{n-1}, f_{m+1}=\sum_{l=0}^{n} J_{l m-(m+1), n-l} \zeta^{l m-(m+1)} w^{n-l}, \\
& f_{m+2}=\sum_{l=0}^{n} J_{l m-(m+1), n-l} \zeta^{l m-(m+2)} w^{n-l}, \ldots
\end{aligned}
$$

$f_{0}$ is none other than the characteristic polynomial of $R$, i.e. $\sum_{\beta=1}^{n}\left(w-b_{\beta} \zeta^{m}\right)$. Let us substitute $(43)$ into $f(w, \zeta)=0$. The coefficient in $\zeta^{m n-m-2}$ gives

$$
q_{\alpha}=\frac{-f_{m+2}\left(b_{\alpha}, 1\right)}{\frac{\partial}{\partial w} f_{0}\left(b_{\alpha}, 1\right)}=-\sum_{l=2}^{m} J_{(l-1) m-2, n-l} b_{\alpha}^{n-l} / \prod_{\beta \neq \alpha}\left(b_{\alpha}-b_{\beta}\right)
$$

and

$$
H=-\sum_{\alpha=1}^{n} a_{\alpha} \sum_{l=2}^{n} J_{(l-1) m-2, n-l} b_{\alpha}^{n-l} / \prod_{\beta \neq \alpha}\left(b_{\alpha}-b_{\beta}\right)
$$

For each $l$, the Hamiltonian depends on $J_{k l}$ with only one value of $k$ : $k=(n-l-1) m-2$. Thus the following holds :

Theorem. $\theta_{k l}$ depend on $x$ thus: if $k=(n-l-1) m-2$ then

$$
\theta_{k l}=-\sum_{\alpha=1}^{n} \frac{a_{\alpha} b_{\alpha}^{l}}{\prod_{\beta \neq \alpha}\left(b_{\alpha}-b_{\beta}\right)} x+\theta_{k l}^{0}
$$


if $k<(n-l-1) m-2$ then

$$
\theta_{k l}=\theta_{k l}^{0},
$$

$\theta_{k l}^{0}$ are constant.

\section{References}

1. Gelfand, I. M., Dickey, L. A. : Funct. Anal. Appl. 13, 8-20 (1979) (Russian)

2. Gelfand, I. M., Dickey, L. A. : Usp. Mat. Nauk 30, 67-100 (1975) (Russian)

3. Gelfand, I. M., Dickey, L. A. : Funct. Anal. Appl. 10, 18-25 (1976) (Russian)

4. Gelfand, I. M., Dickey, L. A. : Funct. Anal. Appl. 10, 13-29 (1976) (Russian)

5. Dubrovin, B. A., Matveev, V. B., Novikov, S. P. : Usp̀. Mat. Nauk 31, 55-136 (1976) (Russian)

6. Dubrovin, B. A. : Funct. Anal. Appl. 11, 28-41 (1977) (Russian)

7. Kuperschmidt, B. A., Wilson, G. : Modifying Lax equations and the second Hamiltonian structure, Preprint

8. Drienfeld, V. G., Sokolov, V. V. : Korteweg-de Vries Equation and simple Lie algebras. (to be published)

Communicated by A. Jaffe

Received June 2, 1980 in revised form January 30, 1981 